\title{
The walkthrough method: An approach to the study of apps
}

\section{Ben Light", Jean Burgess and Stefanie Duguay. \\ (Please see end of paper for author affiliations, contact details and biographies.)}

\begin{abstract}
Software applications (apps) are now prevalent in the digital media environment. They are the site of significant sociocultural and economic transformations across many domains, from health and relationships to entertainment and everyday finance. As relatively closed technical systems, apps pose new methodological challenges for sociocultural digital media research. This paper describes a method, grounded in a combination of science and technology studies with cultural studies, through which researchers can perform a critical analysis of a given app. The method involves establishing an app's environment of expected use by identifying and describing its vision, operating model, and modes of governance. It then deploys a walkthrough technique to systematically and forensically step through the various stages of app registration and entry, everyday use, and discontinuation of use. The walkthrough method establishes a foundational corpus of data upon which can be built a more detailed analysis of an app's intended purpose, embedded cultural meanings, and implied ideal users and uses. The walkthrough also serves as a foundation for further user-centred research that can identify how users resist these arrangements and appropriate app technology for their own purposes.
\end{abstract}

\section{Keywords}

Digital methods, apps, mobile media, qualitative methods, walkthrough, cultural studies, STS

\footnotetext{
* Author for correspondence and lead author.
} 
"So I guess the tie means that you used some form of protection."

This remark was made during one of our digital methods workshops, where we were teaching app analysis to some of our colleagues and $\mathrm{PhD}$ students. The participant was commenting on the icons used for reporting sexual activity within the menstruation-tracking app Clue (Figure 1). In our discussion, participants raised points about navigational features and their symbolic qualities, which shaped how they viewed the app. Within this screen, the complexity of sexual activity is reduced to four simple icons indicating binary predicaments: sex is either protected or unprotected and, if problems arise, they are due to either a high sex drive or withdrawal from intimate contact. Through oversimplification, the app's presentation of options obscures how safer sex practices can include varying levels of protection, and how individuals can encounter a range of sexual challenges that encompass far more than just having too much or too little sex. The icons also reinforce cultural norms of heterosexuality and elide the possibility of trans identities. Assuming that the user identifies as female and chooses male sexual partners, the app uses a symbolically male clothing item - the tie - that can be read as carrying moral undertones, indicating that protected suitors are prim and proper gentlemen. In this paper, we set out the method that we used in this workshop for the rigorous and systematic study of apps, and that allowed nuanced discussions such as these to emerge from it: the walkthrough method. 


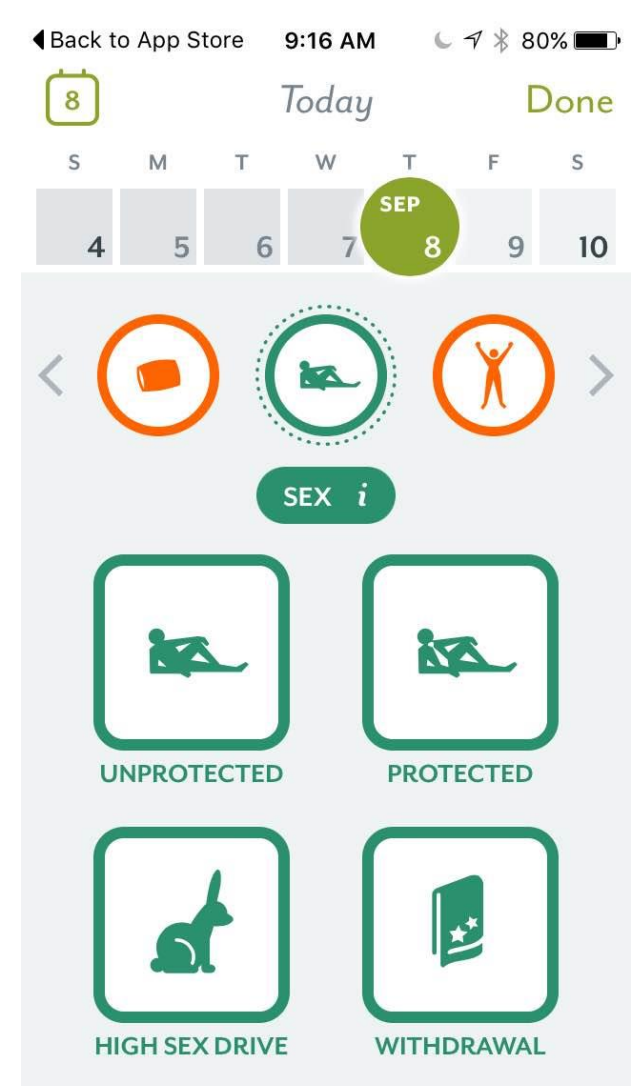

Figure 1. Screenshot from menstruation-tracking app Clue

The walkthrough method is a way of engaging directly with an app's interface to examine its technological mechanisms and embedded cultural references to understand how it guides users and shapes their experiences. The core of this method involves the step-by-step observation and documentation of an app's screens, features, and flows of activity-slowing down the mundane actions and interactions that form part of normal app use in order to make them salient and therefore available for critical analysis. The researcher registers and logs into the app, mimics everyday use where possible, and discontinues or logs out while attending to technical aspects, such as the placement or number of icons, as well as symbolic elements, like pictures and text. This process is contextualised within a review of the app's vision, operating model, and governance. While similar techniques are deployed in User Experience Design and vernacular contexts like technology reviews, the interpretative aspects of the method as we describe it here are underpinned by specific theoretical frameworks: science and technology studies (STS) and cultural studies supply the analytical power to identify connections between these contextual elements and the app's technical interface.

This paper works through the various components of the method, first discussing challenges for app research, the utility of a walkthrough technique, and how we combine STS and cultural studies as a lens for app analysis. We describe how to identify the app's vision, operating model, and governance as investigative work that stakes out the app's environment 
of expected use - how app provider anticipates it will be received, generate profit or other forms of benefit, and regulate user activity. This work to establish the environment of expected use can be done in preparation for the step-by-step technical walkthrough or it can be done alongside the walkthrough process to illuminate the intentions behind particular features and functions ${ }^{1}$. We then explain the process of walking through different phases of app use, and highlight points of interest that may be common across a range of research contexts. We conclude with methodological and ethical considerations, explaining how this approach fits within a toolkit of traditional and digital methods.

Working together on a collaboration around dating and hookup apps, we developed the walkthrough method out of our engagement with the current digital methods literature and discussions with colleagues calling for a way to unite STS approaches of tracing technological systems with cultural studies techniques for recognising discursive and symbolic representations. It has proved essential in our own analyses, allowing for the deep interrogation of apps, such as investigating how Ashley Madison's profile features interact with bots (Light, 2016), exploring the use of apps for engaging with public sexual cultures (Light, forthcoming) and assessing how Tinder's connection with Facebook builds a sense that its users are authentic (Duguay, 2016). The method has also been implemented in comparisons across apps, allowing us to develop a comparative typology of mobile dating and hookup apps (Duguay et. al., forthcoming) as well as contrasting Vine and Instagram's creative features (Duguay, 2016).

We have further refined and elaborated the method through our experience conducting and garnering feedback from a series of research methods workshops involving more than 250 academics and graduate students working across a number of social science and humanities disciplines. These workshops included practical exercises where participants deployed the walkthrough method to analyse apps across domains including gaming, music, health, ridesharing, and microtasking. This process of reflection and refinement has shaped the method into an approach that is now adaptable - in whole or in part - to a range of research questions and agendas. While we invite researchers to apply the walkthrough flexibly and in conjunction with other methods, here we present all its elements sequentially for the sake of coherence and comprehensiveness.

\section{Apps and their methodological challenges}

The increased prominence of apps from around 2008 is often associated with the introduction of Apple's iPhone, iOS and App Store - and indeed, the age of the smartphone 
has proceeded in lockstep with the appification of the digital media environment and the decline of the open web. However, software applications, from where the more generic term 'app' originates, have existed for much longer. Understood in the sense of 'software applications', apps are a subset of computer programs: they are computer programs that solve particular, often singular, user needs - originally, business needs (Pressman, 2005). We usually think of proprietary distribution platforms, such as the Apple App Store or Google Play Store, delivering apps, but contemporary app developers also create for the web (including the mobile web), using technology such as HTML5 to bypass app stores. Apps are a significant component of digital culture and the digital economy. In 2014, worldwide app revenues were $\$ 34.99$ billion (USD) and are expected to rise to $\$ 76.52$ billion (USD) in 2017 (Stasita.com, 2015).

While there is debate about whether the app model of digital media development represents an open or closed cultural system more generally (Burgess, 2012), apps' technical closure presents empirical challenges to digital media researchers. An app's source code is not often shared publicly, prohibiting researchers from examining its underlying structure or operating code as they would with webpages or software programs with accessible structures and file libraries. Although researchers gather digital data for some apps by querying Application Programming Interfaces (APIs) - protocols allowing the app to interact with other software - these queries often return partial datasets, limited to protect commercial interests (Burgess and Bruns, 2015). Further, the API documentation upon which researchers rely when developing programs for data queries is often incomplete (Uddin and Robillard, 2015) and many apps have APIs that are partially or altogether inaccessible to the public. Commercial apps also protect trade secrets and design architecture, requiring innovative approaches, such as auditing an app's algorithms through experimental scenarios (Sandvig et al., 2014). Further, while this sociotechnical closure creates challenges for accessing data through established digital research methods, automated methods that collect 'big' data or metadata can overlook an app's symbolic elements and users' social interpretations (Rieder and Röhle, 2012).

Scholars in relevant areas of the humanities and social sciences have called for new methods appropriate to this contemporary 'computational turn' (Berry, 2011) - that is, going beyond merely using computational tools to tackle traditional social science questions, and instead developing new concepts and methods to study computational technologies as sociocultural artefacts. In elaborating the notion of digital methods, Rogers (2013) asserts the necessity of using the "methods of the medium": studying society and culture through the 
functions and everyday practices of digital media technologies that remediate and shape sociocultural phenomena. Therefore, analysing an app requires attention to its embedded sociocultural representations as much as its technological features or data outputs, which also have social and cultural influences. This is the intent of our approach to studying apps via the walkthrough method. Our approach incorporates the methods of the medium by inviting the researcher to engage closely with the app, using a step-by-step walkthrough technique that involves progressing through the app's requirements, screens, and activities to understand how it guides users.

\section{Prior uses of walkthroughs}

Walkthroughs are an established genre of vernacular cultural practice, particularly in the consumption and evaluation of cultural goods (Grimes, 2015; Sing et. al., 2000). Walkthroughs, in this vernacular sense, can have pedagogical and commercial value. Examples include traditional infomercials, instructional game walkthroughs, and walkthroughs as key elements of game, app, and software reviews on sites such as YouTube, which hold the potential to educate and persuade target audiences (Lee and Hoffman, 2015; Sing et. al., 2000; Smith and Sanchez, 2015). Vernacular walkthroughs reveal intricate details about the artefact in question, creating a step-by-step narrative of use. Walkthroughs often make explicit the otherwise implicit and (by design) apparently seamless process of engaging with a digital media object - and they can give away hidden affordances and tricks (as in game walkthroughs which can reveal shortcuts and workarounds for wickedly difficult elements of gameplay). Of course, like all cultural texts, walkthrough videos and narratives inevitably reflect the discursive and ideological positions of their producers, and align with their agendas (e.g., performing technical mastery or critical expertise, increasing sales, generating followers).

In more formal academic settings, early uses of the walkthrough as a technique were grounded in software engineering and oriented toward improving the quality of code and user experience (Fagan, 1976). Human-Computer Interaction (HCI) drew from software engineering and formalised "user walkthroughs" as devices to assist in the creation of more usable and useful digital products - in particular by highlighting users' departures from the intended procedures or pathways, and then tweaking the design in response (Lewis et. al., 1990; Nickerson and Landauer, 1997). The walkthrough method we describe here is a significant departure from how similar techniques have been used in such contexts. The walkthrough method we propose is used, not to test whether users respond to an interface in 
the ways its designers intended, but rather to illuminate the material traces of those intensions, and thereby to critically examine the workings of an app as a sociotechnical artefact. It does so by grounding the step-by-step technique in a combined framework of STS and cultural studies that allows for identifying the technological mechanisms that shape - and are shaped by - the app's cultural, social, political, and economic context.

\section{Conceptual Framework}

The walkthrough method as we use it is grounded in the principles of Actor-Network Theory, as a specific aspect of Science and Technology Studies. Actor-Network Theory (ANT) foregrounds a relational ontology according to which sociocultural and technical processes are mutually shaping (Callon, 1989; Latour, 2005). Among the actors configured in relation to a particular technology, ANT differentiates between intermediaries and mediators, which can additionally be human or non-human (Latour, 2005). Intermediaries pass meaning along unchanged throughout a network of relations while mediators are transformative - they alter the meaning or circumstances within a system. In the case of apps, user interfaces and functions are therefore understood as non-human actors that can be mediators. For example, a dating app can take a simple piece of information about a person, such as an affinity for the outdoors, and transform its meaning by adding a fitness-related emoji to the user's profile, implying healthiness due to the program's associations with particular hobbies and symbolic repertoire for indicating such associations.

Another way of understanding the influence of non-human actors is through consideration of a technology's materiality and the affordances it extends. Bucher and Helmond (2017, in press) note that affordance theories have progressed from understanding affordances broadly as behaviours that an environment offers or constrains (Gibson, 2015) to actions guided by a technology's design (Norman, 1988) and eventually acknowledging the role of social and material influences on how users perceive actions they can take in relation to a technology. The walkthrough examines affordances at multiple levels of scale (McVeigh-Shultz and Baym, 2015), from the app's buttons to its interaction with operating systems, hardware, structures of connectivity (e.g., wifi), and other apps in its extended environment. Attention to materiality identifies physical interactions encouraged by the app, from Tinder's thumb swiping for selecting matches to WeChat's phone shaking to find chat partners. Being mindful of apps' material influences allows the researcher to place oneself in the user's position and imagine the range of affordances the user perceives. 
Since the technological architectures of apps are a kind of infrastructure, they can be examined in a similar fashion as in prior research on infrastructural systems. Star (1999) describes how infrastructure is invisible in the sense that when individuals pour a glass of water, they do not consider the intricate plumbing systems involved in its delivery. Similarly, app users may overlook the icons and screen sequences integral to their everyday activities. By definition, apps more closely resemble 'platforms' as closed and controlled systems, which are often commercially owned and organise activity within a specific software program (Plantin et al., 2016). However, the "infrastructuralization of platforms" (Plantin et al., 2016, p. 9) as they grow and interconnect means that apps can be examined using infrastructure studies techniques. Star (1999) suggests bringing an "ethnographic sensibility"2 (p. 383) to closed-off or hidden systems through multiple approaches including literary analysis, observations, and systems analysis. The central walkthrough technique of stepping through the app incorporates elements of ethnography through observation and generating field notes. Establishing the app's environment of expected use, requires digging through its related materials and ancillary media. The walkthrough draws on previous approaches to infrastructure ethnographies to make an app's system of actors visible for analysis.

While focused on interactions among actors, STS scholars also noted cultural influences within technological systems. These are identified as master narratives (Star, 1999) as an arrangement of actors that declares a particular understanding, such as medical forms reinforcing gender binaries through checkboxes for only male or female patients. In tracing struggles between users and technology creators, Pfaffenberger (1992) identified that technologies serve the cultural aspirations of their creators, who often accrue power by oppressing particular groups. Technologies are deployed with symbolism and rituals to reinforce these aims. For example, a menstruation-tracking app may be developed within a male-dominated technology industry whereby men succeed through women's oppression. Within this cultural context, its designers do not think twice about symbolising pre-menstrual syndrome through a whirlwind icon intimating that women act as chaotically as natural disasters several days each month. Through this recognition of cultural influences in technological systems, we interlock STS concepts with cultural studies approaches to identifying and deconstructing a technology's cultural discourses.

The walkthrough method builds on scholarship in cultural studies that, similarly to the aspects of STS we draw on above, attends to how technologies shape culture while simultaneously being a product of it; considering also the symbolic or representational elements of cultural objects in combination with the technological or material ones. In 
Raymond Williams' (1974) cultural analysis of television at a time when, like mobile media, it was still fairly new but had become firmly embedded in and representative of institutional regimes of power, he underscored how technology neither determines society's trajectory nor is it symptomatic of social change. Instead, technologies are designed, experienced, and further developed within a culture that shapes and is influenced by them. Recognition of this mutual shaping is integral to the walkthrough, which takes the researcher through a process of identifying cultural values embedded in app features and imagining how these features, in turn, seek to reinforce values among users.

The walkthrough's close engagement with app technology mobilises the existing methodological toolkit of cultural studies, particularly as it is concerned with studying material culture and everyday practices of technology consumers. Du Gay et al.'s (2013) 'circuit of culture', applied originally to study the Sony Walkman as material artefact and site of cultural struggle, identifies the sociocultural processes surrounding an artefact. It regards "how [a technology] is represented, what social identities are associated with it, how it is produced and consumed, and what mechanisms regulate its distribution and use" (p. xxxi). Examining an app's environment of expected use begins to uncover these elements and their direct presentation to users becomes apparent during the step-by-step walkthrough. For example, Tinder's promotional videos tend to feature actors in their twenties participating in youthful activities (e.g., road trips, rooftop parties). These efforts to target younger users are realised in the app's subscription screen offering a lower fee to younger users and the preferences screen sorting older users into a broad '55+' category. The circuit of culture provides a frame from which to identify embedded cultural values while walking through the app's interface.

While digital media scholars have begun identifying cultural influences within communications technologies, the walkthrough method is tailored to apps. Studies of websites have identified how features, such as drop-down menus, shape users' identity expression (Nakamura, 2002). Others have focused on commercial influences steering the development of technology practices, such as Nokia's role in shaping early multimedia messaging practices (Lillie, 2012). Recent studies of social media platforms have identified how platform companies' economic and political interests guide a platform's development (van Dijck, 2013), such as by analysing Mark Zuckerberg's public rhetoric about Facebook (Hoffman et al., 2016). The walkthrough method extends these approaches to consider how qualities specific to apps, such as geolocative features, mobile access, and distribution 
through apps stores, also feature in cultural struggles among a technology's economic, political, and social players.

The need for a new approach is evident in related methods that involve similar considerations but do specify how to interrogate an app's technological architecture. Recent interview-based methods in media and cultural studies have adopted participant-led platform explorations. These include scrolling back through a participant's Facebook Timeline (Robards and Lincoln, in press) or "media go-alongs" where users respond to questions as they interact with an app (Jørgensen, 2016). While these approaches highlight app features, understandings are interpreted through users, who place their lens of experience upon these qualities. While the walkthrough method can similarly be conducted alongside users, its standard application simply involves the researcher examining what the app contributes to users' interactions with it. This more closely resembles components of Critical Technocultural Discourse Analysis (CTDA), which examines an artefact's interpellation, or calling out, of certain identities according to its embedded ideologies (Brock, 2012). Whereas CTDA examines a technology's interface alongside user practices, the walkthrough method focuses on interface elements and their connection to the app's environment of expected use. It provides a systematic approach to identifying cultural discourses that shape and are perpetuated by interface elements, which allows for integration with frameworks like CTDA or mixed methods studies.

In combining compatible concepts and approaches from STS and cultural studies, the walkthrough method is representative of a broader shift to the dual consideration of technology and culture in response to the 'computational turn' (Berry, 2011). The following sections outline the method's processes of data collection and analysis, establishing an app's context and then walking through the program itself to identify key technological mechanisms of cultural meaning.

\section{The environment of expected use}

This part of the walkthrough points researchers toward pivotal aspects of an app's context for analysis in conjunction with its technological architecture. It draws from van Dijck's (2013) recognition that beyond users, content, and technology, researchers must also account for the socioeconomic and cultural aspects of platforms. Examining an app's vision, operating model, and governance allows researchers to understand how an app's designers, developers, publishers and owners expect users to receive and integrate it into their technology usage practices. 
Vision

An app's vision involves its purpose, target user base, and scenarios of use, which are often communicated through the app provider's organisational materials. This is not just a question of considering users as markets in the capitalist sense (though this may be important), it also examines conceptions the app conveys about activities it is supposed to provide, support or enable (Light et. al., 2008; Papacharissi, 2009; Light and Mcgrath 2010; Light, 2014). For example, Tinder is presented as being for those interested in relationships between two people, referencing a particular view of monogamous intimate relations. In contrast, Squirt, a hook-up app for men who have sex with men, highlights its functionality for communicating with multiple partners at once, disregarding monogamous relationship norms. An app's vision tells user what it is supposed to do and, by extension, implies how it can be used and by whom. While users often expand upon or subvert this, understanding the app's original vision provides a baseline for identifying user appropriation.

Apps disseminate their vision through numerous means. App stores may moderate communications about an app's vision, as they regulate access and use through tight control of app development (Goggin, 2011), while many apps have webpages further elaborating their product's niche. For example, Clue's app store description pragmatically explains its purpose as a 'period tracker' while its webpage declares it as "Beautifully scientific!" alongside a photo of presumed data experts gathered around a phone with serious expressions. Through this imagery, the company differentiates its app by appealing to users who desire empirical rigor when tracking their periods. It also ties into longstanding traditions of medicalising women's bodies, subjecting them to the scientific gaze (Bartky, 2003). Other sources for data collection include company blogs, marketing materials, press releases, and public statements from representatives of the organisation that provides the app. These often establish an app's discursive and symbolic representation (e.g., logos, colour schemes, images), which is carried through to its technical interface. Clue's scientific theme gives rise to clean and streamlined aesthetics that lend some explanation to the app's simplistic icons.

\section{Operating model}

An app's operating model involves its business strategy and revenue sources, which indicate underlying political and economic interests. Revenue generation may involve payment for the app or in-app purchases, permitting access to additional functions (e.g., 
rewind/redo in Tinder), increased levels of access (e.g., unlimited profile viewing in Squirt) or tokens for increased engagement, such as RSVP's exchange of stamps for messaging abilities. Since many apps allow access to services in exchange for personal data that can be sold to advertisers and data miners (van Dijck, 2013), revenue generation may not involve monetary exchange. Both levels of access and the quality of user experience can depend on how much data users provide. Such an exchange commences during registration, with app companies collecting basic information (e.g., email, name, birth date) and escalates as users encounter features requiring more data (e.g. location, connections to social media platforms). Apps may cultivate multi-sided markets, garnering revenue from in-app advertising and purchases as well as partnerships with other platforms (Nieborg, 2015). While some apps, such as those produced by governments or non-profits, have little commercial interest, these still receive resource support to operate, such as through public funding or donations. In-app economies also function among users; for example, sharing lives in gaming apps or forms of recognition that generate social and cultural capital.

App-generated materials, technology industry sources, and public market information are all useful sources for determining an app's operating model. The app's price in app stores, in-app purchase menus, employee recruitment materials, press kits, and other documents about the app provider all provide indications of the app's profit or income generating mechanisms and about the app provider itself. For commercial apps, LinkedIn and company databases like Crunchbase provide information about a company's employee base, funding/investors, and recent acquisitions. Business media outlets, such as Forbes, often interview companies about their business expansion plans. If a company is publicly traded, articles about its Initial Public Offering (IPO) and official documents, such as its SEC filing (financial statements lodged with the U.S. Securities and Exchange Commission) provide a wealth of information about revenue and future projections.

\section{Governance}

An app's governance involves how the app provider seek to manage and regulate user activity to sustain their operating model and fulfill their vision. Governance is reflected in the app's rules and guidelines, which place boundaries around the types of activity that users are able to conduct, and even the types of users allowed on an app. This regulatory work may also be hard coded into an app's features, such as through text recognition mechanisms that disallow posting about certain topics. Governance may expand from simply managing user 
activity to enforcing norms and values. Apps may enlist users in enforcing governance through mutual surveillance facilitated by reporting systems.

Governance is often enacted through formal Terms of Service (TOS) documents and informally by encouraging users to behave in ways that align with the norms of the ideal forms of user the app is targeted at. The length, complexity, and nature of TOS indicate conceptions of governance, especially when studied over time using tools like the Internet Archive's Wayback Machine. Formal policies may provide information about the ownership and applications of user data, privacy and safety matters, and expected community standards. Copyright licenses tend to vary across sites and require attention since they often defy user expectations of the app provider's right to their content (Fiesler, Lampe, and Bruckman, 2016). Similar matters might also be found in a Frequently Asked Questions (FAQ) section, which generally offers informal governance by articulating community norms and preferred user practices. Popular and technical media often capture quotes from app provider representatives about expected user conduct. Finally, the platform through which the app is delivered may act as a governance mechanism, whether this involves an app store's terms or the legal status of the activity the app enables in a particular geographical region.

\section{The technical walkthrough}

The technical walkthrough is the method's central data-gathering procedure. It involves the researcher engaging with the app interface, working through screens, tapping buttons, and exploring menus. Walking through the app requires the researcher assume a user's position while applying an analytical eye to the process of acquiring the app, registering, accessing features and functionalities, and discontinuing use. The researcher adopts an STS approach of systematically tracing key actors, such as icons and purchase buttons, producing a collection of data by generating detailed field notes and recordings, such as through screenshots, video recordings of the phone screen, and audio recordings of one's thoughts while conducting the walkthrough. This involves attention to the app's materiality, including the actions it requires and guides users to conduct, and imaging how users would perceive these as affordances or constraints. It also involves drawing on cultural research skills in textual and semiotic analysis, recognising indicators of embedded cultural discourses, such as how the app constructs conceptions of gender, ethnicity, ability, sexuality, and class.

An app's technical and cultural influences are conveyed through mediator characteristics, which provide indications of how the app seeks to configure relations among 
actors, such as how it guides users to interact (or not) and how these actors construct or transfer meaning. Drawing on ANT's notion of mediators, an app's mediator characteristics transform meaning through the interactions they invoke. They are embedded with culture because their meanings exist in reference to cultural texts and understandings that exist outside the app. Mediator characteristics may include:

- User interface arrangement: How the app guides users through activities via the placement of buttons and menus. For example, some buttons like "Report" or "Share" may be smaller or harder to find than others;

- Functions and features: Groups of arrangements that mandate or enable an activity, including pop up windows, compulsory fields, and requests made by the app to link with other user accounts;

- Textual content and tone: More than instructions, this includes text embedded in user interfaces, such as the order of drop down menu options or the categories available (e.g., sexual identity categories on dating apps) and their discursive power to shape use;

- Symbolic representation: A semiotic approach to examining the look and feel of the app and its likely connotations and cultural associations with respect to the imagined user and ideal scenarios of use. This may involve considerations regarding branding, colour, and font choices.

As researchers traverse the app, interrogating mediator characteristics renders visible aspects of the interface that may otherwise blend into the background of everyday use.

While apps present a range of uses, we have grouped these into three common stages for data generation through documentation and analysis during the walkthrough. These stages include: registration and entry; everyday use; and suspension, closure and leaving. While these categories are not discreet (e.g., registration fields often construct a profile that features in everyday use), we present them separately for the sake of discussion.

\section{Registration and entry}

This is often the starting point of a walkthrough, involving description and analysis of how a user sets up an account for a particular app. Initial contact with an app may vary as they may be part of a larger infrastructure. Some may require registration through a desktop computer while others are only available through mobile technology and require registration through a tablet or phone. Squirt allows users to register via the website and subsequently 
sign into mobile site in a phone's browser, since it is not vetted by app stores. Users can, however, register via the mobile app and choose to only access it through its reduced mobile functionality. The mobile version emphasises proximity to other users and facilitates quick encounters while the desktop version allows for greater interactivity in Squirt's online forums. Choosing between these routes presents different app mediators and alters the user experience.

Registration can occur through other mechanisms, such as automatic functions by which an app calls out to partner services or retrieves existing user content. For example, connecting with Facebook is a compulsory form of identity verification on Tinder. Where this occurs, it is necessary to consider the nature of the connection and what elements (e.g., usernames, passwords, content) of the connected service become actors within the registration process. Other examples of common registration steps include requests for access to a user's personal contacts, photographs, or location. If a profile is required, as is the case with some games, the registration and entry process may be as simple as installation or pointing a browser to a URL.

The app's expected use is often strongly communicated during registration. The vision may be stated in welcome screens and the operating model becomes salient in offers for premium versions. Registration is also a stage where an app generally communicates its governance, including 'terms of use' screens that require users to click "I agree." Preferred user practices go hand-in-hand with subtle suggestions about the ideal user, often depicted in 'how to' screens that demonstrate app use.

\section{Everyday use}

This stage refers to activities that registered users regularly engage in. This part of the walkthrough method focuses on recording the functionality, options, and affordances that the app provides to users. This may require creating multiple profiles to engage with algorithms tailored to certain user groups. Not all functions may be easily accessible to a researcher who does not wish to or is not able to interact with other users, pay money, or execute certain activities (e.g. spending hours to unlock a level in a game). However, walking through even the app's basic functionality provides a sense of what activities it enables, limits and guides users toward. Paying attention not only to the app's features but also to the flow of activity (i.e. the order of screens and functions) provides valuable data. To give some sense of what considerations during this stage, we offer examples from apps with contrasting purposes: the hook-up app Squirt and the game Pet Rescue Saga (Figure 2). 


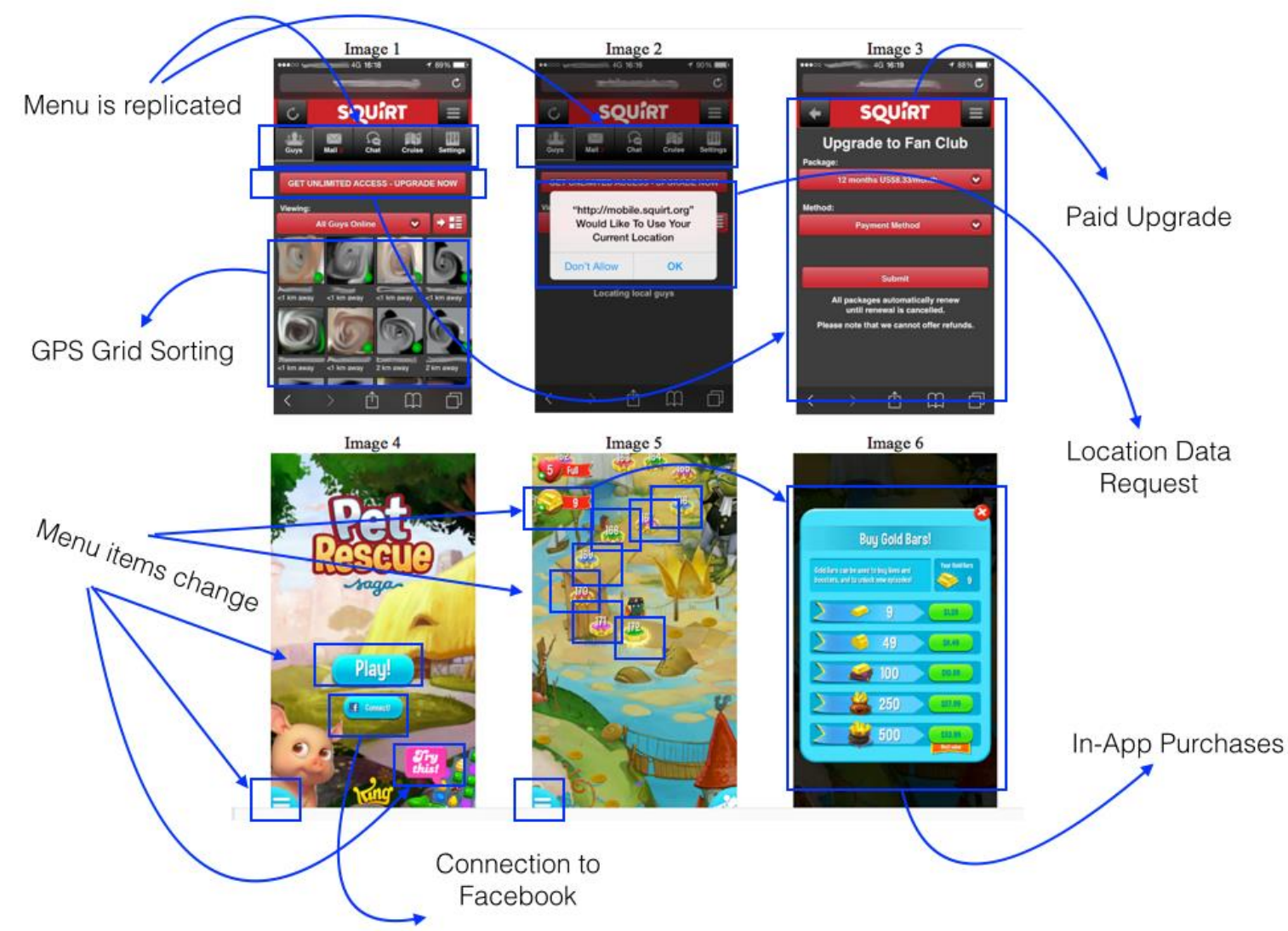

Figure 2. Comparison of Squirt and Pet Rescue Saga

The app's menu can be used initially to trace mediators. All apps have a menu system but this may not be presented in an obviously structured fashion. Considering interface arrangement, it is possible to clearly see Squirt's structuring as it uses tightly organised navigation text and images to guide users. The navigation structure remains the same at deeper levels (images 1 and 2). Menu icons are also key features that can symbolise cultural representations. Squirt's inclusion of a 'cruising' tab with a treasure map references a history of gay men meeting covertly in semi-public spaces (Mowlabocus, 2008). These icons and the app's aesthetic include red as a colour of excitement but grey dominates. This provides a serious tone, emphasising the app's streamlined functionality that digitally mediates cruising, shifting it into a more precise, private activity than its offline practice.

In contrast, Pet Rescue Saga is less structured and is playful in its aesthetics and symbolism. Menu items are not immediately obvious and change depending upon the user's position within the app (images 4 and 5). During a walkthrough, the researcher may use the menu to anchor exploration, tapping on each item and following its associated activities. 
Depending on the research focus, it may instead be relevant to anticipate how users would select menu items and focus on the most common activities.

Since attending only to static interface elements risks missing important points of mediation, work is needed to unearth how and why a set of associations exists. For example, Squirt uses locative grid sorting to organise members' profiles in a geographic fashion so users can identify who is nearby. The app emphasises user geography manually via postcode (if entered) and automatically via a request for access to locative services (during login - see image 2). These aspects cannot be discerned without further engagement as the researcher performs the walkthrough and allows the app to present use requirements or options. Following these activity flows can help to identify how the app mediates users in cultural spaces. Grid sorting within hookup apps for men seeking men can create a co-presence among users that increases their sense of belonging even when physically situated in heteronormative public spaces (Blackwell, Birnholtz, and Abbott, 2015). In Pet Rescue Saga, connecting with friends via Facebook enables the ability to request lives and share them with other players - a key in-app currency but a functionality that can impose on friend networks if accompanied by multiple automatic requests. These examples illustrate how the researcher must follow some activity flows to better understand an app's navigation and potential role in users' lives.

\section{App suspension, closure, and leaving}

Temporarily or permanently leaving apps is complicated for users and has implications for developers (Light and Cassidy 2014; Brubaker et al., 2014; Karppi, 2011). Non-use can range from logging out to hiding profiles and removal of user data. Different apps attempt to retain user engagement in multiple ways. For example, Facebook seeks to keep users engaged by offering opportunities to maintain one's data in the space rather than have it removed (Light and Cassidy 2014; Karppi, 2011). Deleting the Facebook app may seem straightforward: on an iPhone, users deleting the app are simply advised that 'Deleting Facebook will also delete all of its data.' However, this is not fully accurate, as this action deletes data from the user's device but not from Facebook's database. Users must log into Facebook's website to go through the process of data removal, during which they are often prompted to reconsider deletion. Facebook enrols affective content and tone, telling users they 'will be missed by friends' and performs symbolic persuasive work, displaying images of friends to be lost while offering a seven-day reactivation option. 
The process of account suspension, closure or leaving may not always represent a total break of the relationship between user and app. The walkthrough may reveal ways that leaving invites new modes of participation. For example, once Tinder users left swipe to discard a potential match, they will not be shown that user's profile again. However, users may delete their account and register anew to reset the database of possible matches. Walking through aspects of apps that allow users to disconnect can provide insights into how apps seek to sustain use, retain value from users even after they leave (e.g. by continuing to sell their data), and mitigate features that may otherwise dissuade use.

\section{Assessing evidence of unexpected practices}

Although the walkthrough method (when not combined with other methods) does not involve interviews with or observations of users, exploring the app's interface and environment of expected use may uncover evidence of unexpected user practices. This moves beyond identifying user deviations from the designer's original vision (e.g., choosing not to complete certain profile fields) to recognising user-led activities, artefacts, or services associated with the app. These include developing and using third-party apps to extend functionality, social media to develop and critique normative user practices, and code to hack and distort an app's functionality. Tinder's unexpected use has involved the development of third-party apps rearranging its interface, art projects critiquing users for treating others like pieces of meat (Maureira, 2014), and code to make male users believe they are chatting with women when they are really talking to each other (Zelenko, 2015). Since acts of technology appropriation can shift power from designers to users (Eglash, 2004), these unexpected uses can re-allocate some control of the app experience to users, creating new purposes for apps, thwarting profit-making strategies, and circumventing governance tactics.

It may be necessary to perform the walkthrough with third-party apps, since they often transform the original app's mediators. For example, Bonfire replaces Tinder's swipeto-match functionality with a grid interface (similar to Squirt) enabling users to see and like many profiles at once. This grid is not sorted by location, as it would be in Squirt, because Tinder relies less on location as a matching criterion even though it uses GPS technology. This differentiation may not have been identifiable through a Tinder walkthrough alone. Bonfire also filters out friends of friends, challenging Tinder's assumption that users with shared friends want to date each other. This indicates that Tinder's vision may be incongruent with social practices, such as the tendency to reveal more intimate information to potential suitors than one would share with Facebook friends. Tracing evidence of unexpected 
practices can provide insight into how users, and even outside developers, reconfigure an app's relations to challenge, extend, and break free from its environment of expected use.

\section{Ethical and methodological considerations}

While some apps pose greater ethical and legal complications than others (e.g. the use of hook-up apps for sex work), the walkthrough method calls for consideration of two particular ethical concerns regardless of the app being examined. First, while the walkthrough avoids interaction with users, there is the possibility that it may disturb users anyway. If a dummy account is created, others may consider this account to be real and attempt to engage with it. We have opted in our research to ignore user attempts at interaction but researchers must assess these situations individually. One may imagine that when walking through a peer support or counselling app, a message of distress or other attempts at interaction would require careful handling. Strategies for managing potential user interactions need to be considered before any empirical work takes place.

Secondly, a great deal of user information, such as users' names, photos, and in-app activity, can be observed and recorded during the walkthrough. If the researcher is not engaging directly with users, they will not have provided informed consent for the use and display of their data. Even if these users could provide consent, it may not always be appropriate to request it (Hammersley and Atkinson, 2007). User data should be treated similarly to data gathered from observation methods, for example, through anonymisation. If it is pertinent to demonstrate arrangements between the app and users, researchers might use their own or dummy profiles, contact users to obtain consent, or rely on publicly available marketing materials. Since it is not always possible to know whether users intend their data to be used outside of its original context, there are ethical issues to consider wherever research protocols result in the re- or de-contextualisation of such information.

The walkthrough method's limitations can be addressed by combining methods or data sources. While walking through an app can provide a sense of user engagement, the walkthrough does not directly collect and analyse user content, activity, or attitudes. App reviews, news articles, and online user discussions can provide supplemental data. For example, discussions on the Tinder subreddit (reddit.com/r/tinder) often include opinions about the latest updates. As mentioned, user interviews and user-led walkthroughs can also provide insight into how individuals engage with a particular app. Some functionality may be a by-product of programming aspects rather than a deliberate developer choice. Interviews with developers in technology-related news can clarify their intentions. Since this method 
does not engage with user content, researchers can pair it with data collection techniques that query APIs or close readings of user data samples. Combining the walkthrough method with other forms of data collection heeds discussions of how digital methods can incorporate other digital and traditional methods to expand and compare datasets (Snee et al., 2015). Since apps are not stabilised artefacts, it may be necessary to conduct the walkthrough multiple times throughout an app's development and updates.

\section{Conclusion}

Apps matter because they reflect our cultural values, bring multiple actors including users, developers, and advertisers into an interaction space, and communicate meanings that shape our everyday practices. The walkthrough method introduces an approach to studying apps that mobilises concepts from STS and cultural studies to examine these arrangements. It enables researchers to identify the app's context, highlighting the vision, operating model, and governance that form a set of expectations for ideal use. By walking through the app's registration, everyday use, and deletion, this technique allows for recognition of embedded cultural values in an app's features and functions. Once an app's intended use is established, user-developed practices, services, and artefacts provide a sense of how individuals resist these intentions. The walkthrough method is versatile and provides foundational analysis of an app, which can be combined with content analysis or interviews to gain further insights into users' application and appropriation of app technology to suit their own purposes.

We developed the walkthrough method to dig deeper into how apps frame users' selfexpression, relationships, and interactions. It provides a systematic approach to thinking through mobile software components that are often overlooked or subsumed in broader analyses of mobile technologies. We have illustrated how even small associations make a difference, such as the influence of a menstruation-tracking app's icon on how users may feel about their sexual partners and practices. The walkthrough method uncovers these associations and makes them available for analysis.

\section{Notes}

1. The entire approach is called the 'walkthrough method' and the step-by-step process is a walkthrough of the app itself.

2. While Star and others sometimes engage with ethnomethodology, this approach draws on ethnographic methods but links them with a theoretical perspective of social action (Dourish and Button, 1998) that is outside the scope of our framework. We invite 
researchers to layer theories into analysis of data collected through the walkthrough method as it suits their research questions.

\section{References}

Bartky SL (2003) Foucault, femininity, and the modernization of patriarchal power:

Sexuality, appearance, and behavior. In: Weitz R (ed) The Politics of Women's Bodies.

Oxford: Oxford University Press, pp.25-45.

Berry DM (2011) The computational turn: Thinking about the digital humanities. Culture Machine 12: 1-22.

Blackwell C, Birnholtz, J and Abbott C (2015) Seeing and being seen: Co-situation and impression formation using Grindr, a location-aware gay dating app. New Media \& Society 17(7): 1117-1136.

Brock A (2012) From the blackhand Side: Twitter as a cultural conversation. Journal of Broadcasting \& Electronic Media 56(4): 529-549.

Brubaker JR, Ananny M and Crawford K (2014) Departing glances: A sociotechnical account of 'leaving' Grindr. New Media and Society 18(3): 373-390.

Bucher T and Helmond A (in press) The affordances of social media platforms. In: Burgess J, Poell T and Marwick A (eds) The Sage Handbook of Social Media. London and New York: Sage.

Burgess J (2012) The iPhone Moment, the Apple Brand and the creative consumer: From "hackability and usability" to cultural generativity. In: Hjorth L, Richardson I and Burgess J (eds) Studying Mobile Media: Cultural Technologies, Mobile Communication, and the iPhone. New York: Routledge, pp.28-42.

Burgess J and Bruns A (2015) Easy data, hard data: The politics and pragmatics of Twitter research after the computational turn. In: Langlois G, Redden J and Elmer G (eds) Compromised Data: From Social Media to Big Data. London: Bloomsbury, pp.93-113.

Callon M (1989) Society in the making: The study of technology as a tool for sociological analysis. In: Bijker WE, Hughes TP and Pinch T (eds) The Social Construction of Technological Systems: New Directions in the Sociology and History of Technology. Cambridge, MA: MIT Press, pp.83-103.

Dourish P and Button G (1998) On "Technomethodology": Foundational relationships between ethnomethodology and system design. Human-Computer Interaction 13(4): $395-432$. 
Du Gay P, Hall S, Janes L, Madsen AK, Mackay H and Negus K (2013) Doing Cultural Studies: The Story of the Sony Walkman [2nd ed]. London: Sage.

Duguay S (2016) LGBTQ visibility through selfies: Comparing platform mediators across Ruby Rose's Instagram and Vine presence. Social Media + Society, 2(2), 1-12.

Duguay S (2016) Dressing up Tinderella: Interrogating authenticity claims on the mobile dating app Tinder. Information, Communication \& Society. Published online before print: 30 March 2016. doi:10.1080/1369118X.2016.1168471

Duguay S, Burgess J, and Light B (Forthcoming) Mobile dating and hookup app culture. In Messaris, P. and Humphreys, L. (Eds.). Digital media, transformations in human communication, [2nd Ed]. New York, Peter Lang.

Eglash R (2004) Appropriating technology: An introduction. In: Eglash R, Croissant JL, Chiro GD and Fouche R (eds) Appropriating Technology: Vernacular Science and Social Power. Minneapolis: University of Minnesota Press, pp.vii-xxi.

Fagan ME (1976) Design and code inspections to reduce errors in program development. IBM Systems Journal 15(3): 182-211.

Fiesler C, Lampe C and Bruckman (2016) Reality and perception of copyright terms of service for online content creation. CSCW'16, 27 February - 2 March, 2016, San Francisco, CA, USA.

Gibson JJ (2015) The Ecological Approach to Visual Perception. New York: Psychology Press.

Goggin, G (2011) Ubiquitous apps: Politics of openness in global mobile cultures. Digital Creativity 22(3): 148-159.

Grimes SM (2015) Little big scene. Cultural Studies 29(3): 379-400.

Hammersley M and Atkinson P (2007) Ethnography: Principles in Practice [3rd ed]. London: Routledge.

Hoffmann AL, Proferes N and Zimmer M (2016) "Making the world more open and connected": Mark Zuckerberg and the discursive construction of Facebook and its users. New Media \& Society. Epub ahead of print 28 July 2016. DOI:

$10.1177 / 1461444816660784$

Jørgensen KM (2016) The media go-along: Researching mobilities with media at hand. Journal of Media and Communication Research 60:32-49.

Karppi T (2011) Digital suicide and the biopolitics of leaving Facebook. Transformations: Journal of Media and Culture 20. Available at: http://transformationsjournal.org/journal/issue_20/article_02.shtml 
Latour B (2005) Reassembling the Social: An Introduction to Actor Network Theory. Oxford: Oxford University Press.

Lee SH and Hoffman KD (2015) Learning the ShamWow: Creating infomercials to teach the AIDA model. Marketing Education Review 25(1): 9-14.

Lewis C, Polson PG, Wharton C and Rieman J (1990) Testing a walkthrough methodology for theory-based design of walk-up-and-use interfaces. In: Proceedings of the SIGCHI Conference on Human Factors in Computing Systems. Seattle, Washington, USA, April 1990, pp.235-242.

Light B, Fletcher G, and Adam A (2008) Gay men, Gaydar and the commodification of difference, Information Technology and People, 21(3), 300-314.

Light B and McGrath K (2010) Ethics and social networking sites: a disclosive analysis of Facebook, Information Technology and People 23(4), 290-311.

Light B (2014) Disconnecting with Social Networking Sites. Basingstoke, Palgrave Macmillan.

Light B and Cassidy E (2014) Strategies for the suspension and prevention of connection: rendering disconnection as socioeconomic lubricant with Facebook. New Media and Society, 16(7): 1169-1184.

Light B (2016) The rise of speculative devices: Hooking up with the bots of Ashley Madison. First Monday, 21(6). http://firstmonday.org/ojs/index.php/fm/article/view/6426/5525

Light B (Forthcoming) Producing sexual cultures and pseudonymous publics with digital networks. In R. Lind (Ed), Race and Gender in Electronic Media: Challenges and Opportunities. London, UK: Routledge.

Lillie J (2012) Nokia's MMS: A cultural analysis of mobile picture messaging. New Media and Society 14(1): 80-97.

Maureira MG (2014) Tender - It's how people meat. Available at: https://vimeo.com/111997940 (accessed 7 July 2016).

McVeigh-Schultz J and Baym NK (2015) Thinking of you: Vernacular affordance in the context of the microsocial relationship app, Couple. Social Media + Society 1(2): 1-13.

Mowlabocus S (2008) Revisiting old haunts through new technologies: Public (homo)sexual cultures in cyberspace. International Journal of Cultural Studies 11(4): 419-439.

Nakamura L (2002) Cybertypes: Race, Ethnicity, and Identity on the Internet. London: Routledge. 
Nickerson RS and Landauer TK (1997) Human-Computer Interaction: Background and issues. In: Helander MG, Landauer TK and Prabhu PV (eds) Handbook of HumanComputer Interaction (2nd ed). Amsterdam: North-Holland, pp. 3-32.

Nieborg DB (2015) Crushing candy: The free-to-play game in its connective commodity form. Social Media + Society 1(2): 1-12.

Norman DA (1988) The Psychology of Everyday Things. New York: Basic Books.

Papacharissi Z (2009) The virtual geographies of social networks: A comparative analysis of Facebook, LinkedIn and ASmallWorld. New Media and Society 11(1/2): 199-220.

Pfaffenberger B (1992) Technological dramas. Science, Technology \& Human Values 17(3): 282-312.

Plantin J-C, Lagoze C, Edwards PN and Sandvig C (2016) Infrastructure studies meet platform studies in the age of Google and Facebook. New Media \& Society. Epub ahead of print 4 August 2016. DOI: 10.1177/1461444816661553

Pressman RS (2005) Software Engineering: A Practitioner's Approach. London: McGrawHill.

Rieder B and Röhle T (2012) Digital methods: Five challenges. In: Berry DM (ed) Understanding Digital Humanities. London: Palgrave MacMillan, pp.67-84.

Robards B and Lincoln S (in press) Making it 'Facebook official': Reflecting on romantic relationships through sustained Facebook use. Social Media + Society.

Rogers R (2013) Digital Methods. Cambridge: MIT Press.

Sandvig C, Hamilton K, Karahalios K and Langbort C (2014) An algorithm audit. In: Gangadharan SP (ed) Data and Discrimination: Collected Essays. Washington, DC: New America Foundation, pp.6-10

Singh M, Balasubramanian SK and Chakraborty G (2000). A comparative analysis of three communication formats: Advertising, infomercial, and direct experience. Journal of Advertising 29(4): 59-75.

Smith P and Sanchez A (2015) Let's play, video streams, and the evolution of new digital literacy. In: Zaphiris P and Loannou A (eds) Learning and Collaboration Technologies. Los Angeles: Springer, pp.520-527.

Snee H, Hine C, Morey Y, Roberts S and Watson H (eds) (2015) Digital Methods for Social Science: An Interdisciplinary Guide to Research Innovation. London: Palgrave MacMillan.

Star SL (1999) The ethnography of infrastructure. American Behavioral Scientist 43(3): 377391. 
Statista.com (2015) Worldwide mobile app revenues from 2011-2017 (in billion US dollars). Available at: http://www.statista.com/statistics/269025/worldwide-mobile-app-revenueforecast/ (accessed 21 August 2015).

Uddin G and Robillard MP (2015) How API documentation fails. IEEE Software 32(4): 6875.

van Dijck J (2013) The Culture of Connectivity: A Critical History of Social Media. Oxford: Oxford University Press.

Williams R (1974) Television: Technology and cultural form. London and New York: Routledge.

Zelenko M (2015) A brilliant Tinder hack made hundreds of bros unwittingly flirt with each other. The Verge. Available at: http://www.theverge.com/2015/3/25/8277743/tinderhack-bros-swiping-bros (accessed 11 September 2016).

\section{Author Biographies}

\section{Jean Burgess - Queensland Unviersity of Technology, Australia}

Dr Jean Burgess (@jeanburgess) is Professor of Digital Media and Director of the Digital Media Research Centre (DMRC) at Queensland University of Technology. Her research focuses on the cultures, politics, and digital methods for studying social and mobile media platforms.

\section{Stefanie Duguay - Queensland Unviersity of Technology, Australia.}

Stefanie Duguay is a PhD candidate in the Digital Media Research Centre at the Queensland University of Technology. She holds an MSc from the Oxford Internet Institute, University of Oxford, and a BASc in Sociology and Psychology from the University of Lethbridge. Her research focuses on the mutual shaping of users and digital technology in the performance of sexual identity. Her work has been published in Information, Communication and Society, the International Journal of Communication, and Disability \& Society

\section{Ben Light - Unviersity of Salford, United Kingdom.}

Ben Light Professor of Digital Society at the Unviersity of Salford. His research concerns people's everyday uses of digital media with a focus on (non)consumption, gender, sexuality and digital methods. His work has been published in Cultural Sociology, Information, 
Communication \& Society and First Monday. His book, Disconnecting with social networking sites, was published by Palgrave Macmillan in 2014. 